Check for updates

Cite this: Mater. Horiz., 2019 , 6. 1675

Received 15th March 2019,

Accepted 26th April 2019

DOI: $10.1039 / \mathrm{c} 9 \mathrm{mh} 00388 \mathrm{f}$

rsc.li/materials-horizons

\section{Reprogramming of cancer invasiveness and macrophage education via a nanostructured antagonist of the TGF $\beta$ receptor $\dagger$}

\author{
Chang Liu, (D) $\ddagger^{\mathrm{ab}}$ Xingjie Hu, $\ddagger^{\mathrm{c}}$ Xiaojiao Li, $\ddagger^{\mathrm{b}}$ Yanfeng Zhou, ${ }^{\mathrm{a}}$ Hui Wang, ${ }^{\mathrm{a}}$ \\ Chunhai Fan (iD *d and Haiyun Song (iD *ab
}

\begin{abstract}
Nanoparticles (NPs) can interact with a large variety of endogenous proteins upon entering a living system. However, the effects of NP adsorption on protein functions and consequent cellular behaviors are poorly understood. Here, a mass spectrometry analysis is applied to delineate a proteome-scale map of the nanodiamond (ND) interactome network in cancer cells, which identifies the transforming

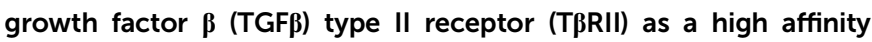
binding partner. Further investigation shows that NDs promote the lysosomal degradation of T $\beta R I$, and thereby suppress the invasion and metastasis of cultured cancer cells, tumor organoids and xenograft tumors via the blockade of the TGF $\beta$ signaling cascade. Significantly, intravenous administration of NDs reduces the recruitment of tumor-associated macrophages (TAMs) and inhibits M2 macrophage polarization in the tumor microenvironment. This study thus reveals NDs as a type of receptor antagonist and suggests their therapeutic effect in cancer treatment.
\end{abstract}

Nanoparticles (NPs) possess exceptional physicochemical properties, which have been extensively utilized for imaging, diagnostic and therapeutic purposes in biomedical research. ${ }^{1-5}$ As a result, the biological consequences of NP exposure have received more and more attention. A diversity of research has highlighted that the interactions between NPs and living systems can initiate a wide range of biological responses. ${ }^{6-8}$ Recent studies including ours have demonstrated that NPs can affect some physiological or pathological processes within the doses that do not induce general cytotoxicity. ${ }^{9-13}$ As examples, fullerene

\footnotetext{
${ }^{a}$ School of Public Health, Shanghai Jiao Tong University School of Medicine, Shanghai 200025, China. E-mail: songhaiyun@shsmu.edu.cn

${ }^{b}$ Shanghai Institute of Nutrition and Health, Shanghai Institutes for Biological Sciences, University of Chinese Academy of Sciences, Chinese Academy of Sciences, Shanghai 200031, China

${ }^{c}$ School of Public Health, Guangzhou Medical University, Guangdong 511436, China

${ }^{d}$ School of Chemistry and Chemical Engineering, and Institute of Molecular Medicine, Renji Hospital, School of Medicine, Shanghai Jiao Tong University, Shanghai 200240, China. E-mail: fanchunhai@sjtu.edu.cn

$\dagger$ Electronic supplementary information (ESI) available. See DOI: 10.1039/c9mh00388f \$ These authors contributed equally.
}

\begin{abstract}
New concepts
Nanodiamonds have been utilized as inert carriers for drugs and biomolecules, yet their interactions with cellular components may confer them more active roles in biological processes, which can be exploited to prevent or correct pathologic activities. Here, the preferential adsorption of nanodiamonds to the TGF $\beta$ receptor is applied to block ectopically activated TGF $\beta$ signaling in tumors. Administration of nanodiamonds attenuates cancer invasiveness and metastasis, and reverses tumor-induced macrophage education, thus expanding the potential of nanodiamonds in cancer therapy.
\end{abstract}

C60 modulates $\mathrm{Ca}^{2+}$ signal transduction via sustaining the kinase activity of CAMKII. ${ }^{14}$ Silica NP exposure hampers tail elongation in Zebrafish via repressing the Wnt signaling cascade. ${ }^{15}$ AuNPs enhance Drosophila lipid anabolism by promoting the PI3K/ Akt/mTOR signaling pathway. ${ }^{16}$ Magnetic iron oxide NPs possess a catalase-like activity and can delay aging and alleviate neurodegeneration. ${ }^{17}$

Nanodiamonds (NDs) are a unique class of carbon NPs that are receiving broad attention for their excellent mechanical and optical properties, large surface area to volume ratio and tunable surface structures. ${ }^{18,19}$ These characteristics of NDs have been utilized for numerous applications in biological and medical research. Due to their stiffness, NDs can be formulated as coating materials in implants to improve the mechanical properties of the composites. $^{20}$ Earlier studies have revealed that incorporated NDs are a type of promising material to promote bone formation in mice. ${ }^{21}$ Fluorescent NDs are ideal probes for long-term imaging in mice and in Caenorhabditis elegans. ${ }^{22,23}$ Moreover, the large surface area to volume ratio and flexible surface modification confer NDs outstanding potential as intelligent nanocarriers for the delivery of drugs, genes and proteins. ${ }^{24,25}$ Although NDs have displayed good biocompatibility in these applications, the potential impacts of NDs on more general cellular behaviors, such as cell differentiation, cell migration and cell communications, are still poorly understood.

NPs interact with endogenous proteins upon entering a living system and form protein corona on their surface, which 
may alter protein conformations and functions, and subsequently trigger a wide range of biological responses including enzymatic reactions, endocytosis and cellular signaling, etc. ${ }^{26-29}$ It has been reported that carbon nanotubes can interact with RNaseA, R-chymotrypsin and soybean peroxidase, resulting in the reduction of their activities via changing their conformations. ${ }^{30,31}$ NDs can interact with the disheveled protein, a key component of the Wnt signaling cascade, and attenuate the activities of Wnt signaling in several biological processes. ${ }^{32}$ In addition, there is an increasing body of evidence that NPs can interact with transmembrane receptors to modulate signal transduction. For example, $\mathrm{TiO}_{2} \mathrm{NPs}$ can disrupt the homophilic interaction of VE-cadherin on the surface of endothelial cells. ${ }^{33}$ AuNPs have been shown to bind to the IgE-FceRI antibody-receptor complex, resulting in the degranulation of rat basophilic leukemia cells and the release of chemical mediators. ${ }^{34}$ Although NDs have displayed large capacity in exogenous protein binding, the interactions of NDs with cellular proteins, the effects of ND adsorption on the activities of these proteins, and the consequences on cellular behaviors are less explored. Given that NDs have great potential in biomedicine, it is highly attractive to address these questions in pathological contexts such as human cancers.

In this study, we employ a mass spectrometric analysis to systematically investigate transmembrane and intracellular proteins that interact with NDs during and after the uptake of NDs by A549 lung cancer cells. The data reveal the TGF $\beta$ receptor II (TRRII) as the most frequently detected transmembrane receptor among the identified ND-interacting proteins. Our further analyses confirm that NDs specifically interact with T $\beta$ RII, but not the TGF $\beta$ receptor I (T $\beta R I)$, and promote its lysosomal trafficking and degradation. Consequently, ND exposure blocks TGF $\beta$ signal transduction, thereby inhibiting cell metastasis in cultured cancer cells and tumor organoids. In a murine model of cancer metastasis, we find that intravenous injection of NDs largely prevents the formation of metastatic tumor nodules in the lung and liver. Significantly, ND administration reduces the recruitment of tumor-associated macrophages (TAMs) and suppresses M2 macrophage polarization in these organs, validating an intrinsic property of NDs in weakening tumor invasiveness and metastasis (Scheme 1).

We characterized the physicochemical properties of the NDs in a recent study, and they possess a primary particle size of about $10 \mathrm{~nm}$, a hydrodynamic size of $191.0 \mathrm{~nm}$, a zeta potential of $41.3 \mathrm{mV}$, and a mixture of ketone, ester, and carboxylic acid functional groups on the surface. ${ }^{35}$ Here we examined their cytotoxicity. NDs are not toxic to A549 cells at or below the concentration of $100 \mu \mathrm{g} \mathrm{mL} \mathrm{m}^{-1}$ in the cell culture medium (Fig. S1, ESI $\dagger$ ).

We adopted a mass spectrometry-based proteomic approach to monitor the dynamic interactions between NDs and cellular proteins in A549 cells. The identified candidate proteins can be categorized into several classes including transmembrane receptors, cytoskeleton proteins, chaperones, enzymes, signal transducers and transcription factors (Fig. 1 and Tables S1-S6, ESI $\dagger$ ). Transmembrane proteins may be the first group of cellular proteins that NPs encounter during their uptake. We found that T $\beta$ RII

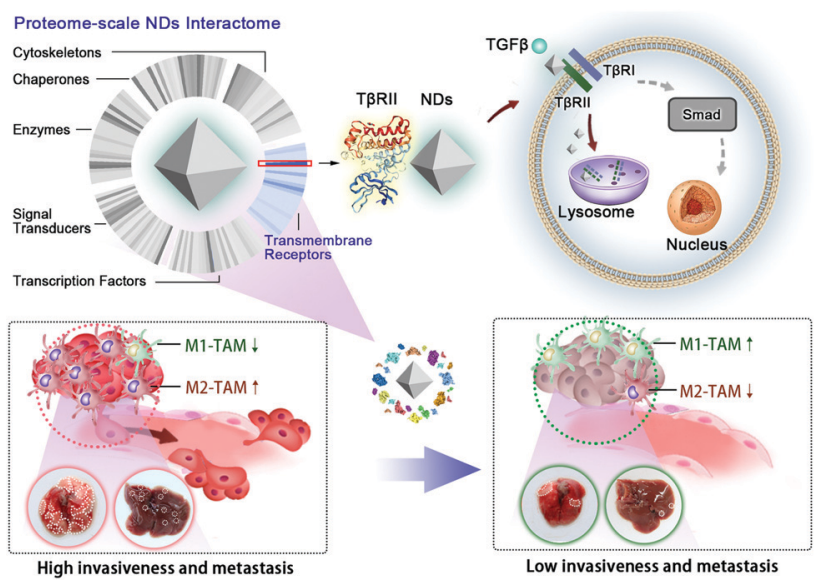

Scheme 1 ND-based receptor antagonist for inhibition of cancer invasiveness and metastasis. A proteome-scale characterization of the ND interactome network in cancer cells reveals T $\beta$ RII as the most abundantly adsorbed transmembrane receptor. NDs promote the lysosomal degradation of T $\beta R I I$ and weaken TGF $\beta$ signaling. Administration of NDs inhibits the invasiveness and metastasis of tumors, and remodels tumor-associated macrophages (TAMs) in the tumor microenvironment.

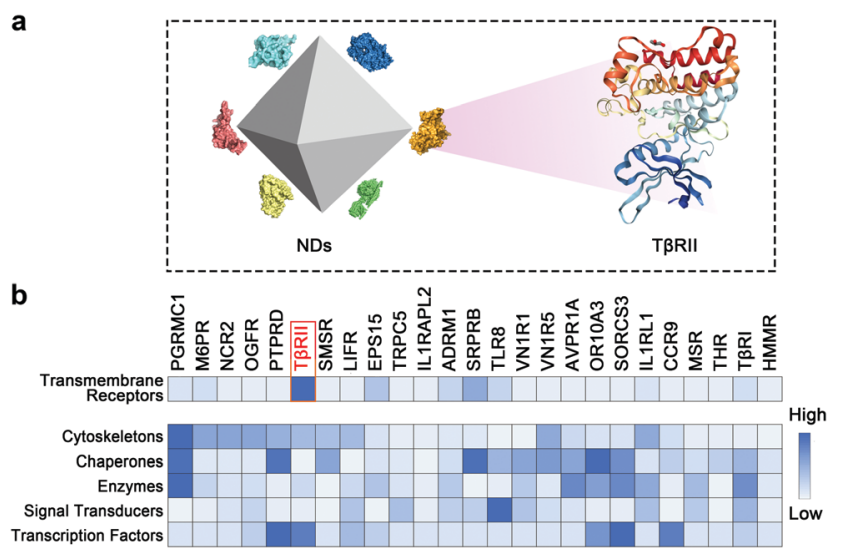

Fig. 1 A proteome-scale map of the ND interactome network. (a) An illustration of ND-protein interaction. (b) Representative ND-interacting transmembrane receptors, cytoskeletons, chaperones, enzymes, signal transducers and transcription factors presented by their relative abundance in the mass spectrometry analysis.

was the most enriched receptor by NDs. A sum of fourteen unique peptides of TRRII was detected by mass spectrometry, covering $26.3 \%$ of its full protein sequence. In addition, TRRI was detected at low abundance (Fig. 1 and Fig. S2, ESI $\dagger$ ). Given that both T $\beta$ RII and T $\beta R I$ are essential components of TGF $\beta$ signaling, these findings imply a connection between NDs and TGF $\beta$ mediated biological functions (Fig. 2a). Similar results were observed when the pull-down assay was monitored by Western blot. Whereas NDs apparently interacted with T $\beta R I I$, the interaction between NDs and T $\beta R I$ was beyond the detection limit (Fig. 2b and Fig. S3a, ESI $\dagger$ ). Therefore, NDs may interact with T $\beta R I$ indirectly, presumably via the dynamic dimerization between TRRI and T $\beta$ RII. ${ }^{36,37}$

After binding to the TGF $\beta$ ligand, T $\beta$ RII and T $\beta R I$ undergo endocytosis and subsequent recycling at a form of early endosome 

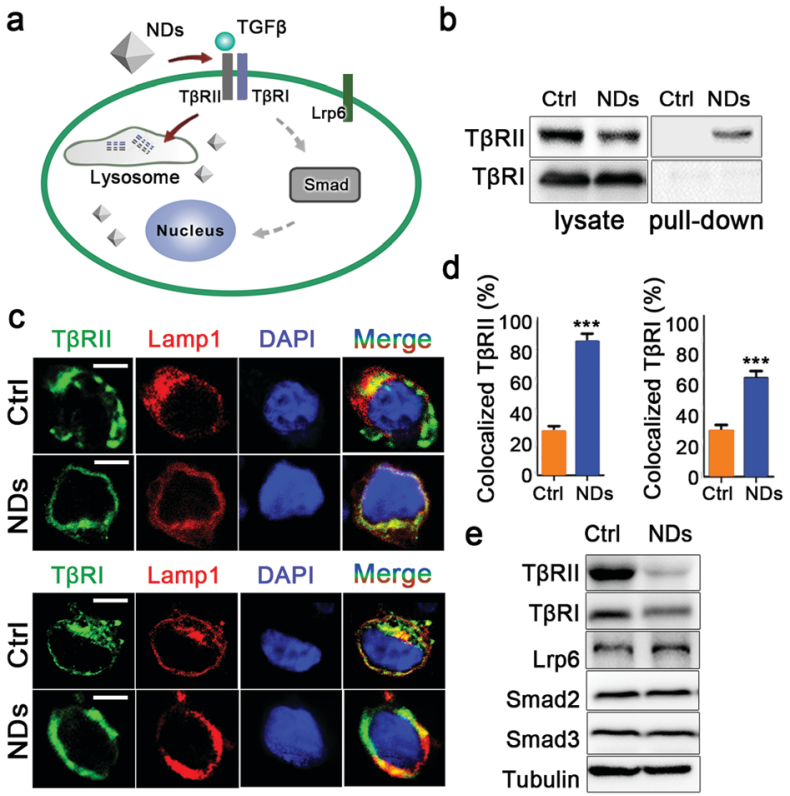

Fig. 2 NDs promote lysosomal degradation of TGF $\beta$ receptors. (a) An illustration for ND mediated interference of TGF $\beta$ signal transduction. (b) The pull-down of TGF $\beta$ receptors by NDs from the lysate of A549 cells. (c) The co-localization of TGF $\beta$ receptors with the lysosomes. Scale bars, $5 \mu \mathrm{m}$. (d) Quantification of the results in (c). Data are represented as mean \pm SD $(n=5)$. Student's $t$-test, ${ }^{* \star *} p<0.001$. (e) The effects of ND uptake on the protein levels of the indicated proteins in A549 cells.

named the recycling endosome. ${ }^{34}$ Since NDs are also internalized via endocytosis, ${ }^{38}$ we investigated whether the interactions of NDs with T $\beta$ RII and T $\beta$ RI affected intracellular trafficking of these receptors. In control cells, only low levels of T $\beta$ RII and T $\beta$ RI were co-localized with the lysosome marker Lamp1, indicating that the basal turnover of TGF $\beta$ receptors occurred in this organelle. ND treatment greatly increased the levels of lysosome-localized T $\beta$ RII. In addition, the lysosomal localization of T $\beta R I$ was also potentiated by NDs (Fig. 2c and d). These results suggest that the adsorption to NDs prevents the sorting of the TGF $\beta$ receptors to the recycling endosomes. Although T $\beta$ RI may not directly bind to NDs, its ability to dimerize with T $\beta$ RII renders it sensitive to cellular uptake of NDs. We followed up to examine the impact of ND uptake on the protein levels of these receptors in A549 cells. As controls, incubation with NDs did not affect cellular levels of TGF $\beta$ signaling components Smad2 and Smad3, or the transmembrane receptor Lrp6. In contrast, it largely eliminated the levels of T $\beta$ RII and T $\beta$ RI (Fig. 2e and Fig. S3b, ESI $\dagger$ ).

Although the cells were extensively washed before the pulldown assay, we examined the possibility that residual endogenous TGF $\beta$ ligand or serum proteins contributed to the above observations. The human embryonic kidney $293 \mathrm{~T}$ cells showed neglectable levels of TGF $\beta$ expression (Fig. S4a, ESI $\dagger$ ), whereas incubation with NDs still decreased the protein levels of TGF $\beta$ receptors in these cells (Fig. S4b, ESI $\dagger$ ). In addition, we cultured A549 cells in a serum free medium. NDs bound to T $\beta$ RII, but not $T \beta R I$, and reduced the protein levels of $T \beta R I I / T \beta R I$ in the absence of serum proteins (Fig. S5, ESI $\dagger$ ). a
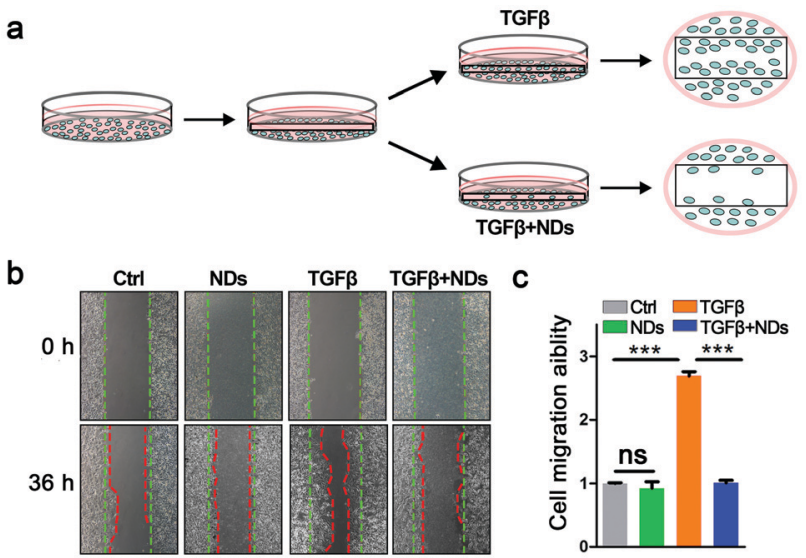

d
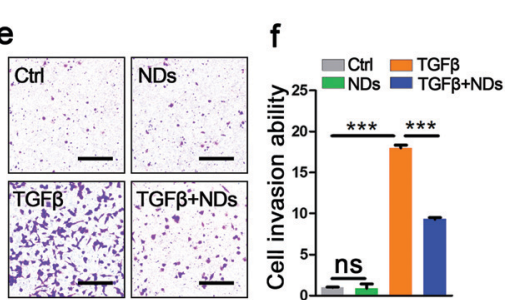

Fig. 3 Effects of NDs on cancer cell metastasis in vitro. (a) A scheme for the wound healing assay. (b) The effects of NDs on cell migration in the absence or presence of TGF $\beta$. The contour of cell migration was labeled by dashed lines. (c) Quantification of the results in (b). Data are represented as mean $\pm \mathrm{SD}(n=3)$. Student's $t$-test, ns: not significant, ${ }^{* * *} p<0.001$. (d) A scheme for the transwell assay. (e) The effects of NDs on cell invasion in the absence or presence of TGF $\beta$. Scale bars, $100 \mu \mathrm{m}$. (f) The quantification of the transwell assay. Data are represented as mean \pm SD $(n=3)$. Student's $t$-test, ns: not significant, ${ }^{* \star *} p<0.001$.

We continued to explore the biological consequences of ND-mediated receptor degradation on TGF $\beta$ signaling activities, which play pivotal roles in cancer invasion and metastasis. The wound healing assay and transwell invasion assay are welldocumented approaches to monitor the abilities of cell migration and invasion. In the wound healing assay, the scratches on confluent A549 cells were gradually recovered, and the addition of the TGF $\beta$ ligand significantly facilitated the migration of cancer cells. Pre-incubation with NDs strongly impeded TGF $\beta$ induced cell migration (Fig. 3a-c). Consistent with the results of the wound healing assay, ND exposure also markedly inhibited TGF $\beta$-induced cell invasion in the transwells (Fig. 3d-f). Furthermore, we examined the expression of TGF $\beta$ target genes including Snail, Nanog, HMGA2, Slug and Mmp-2 during the wound healing assay. Whereas NDs displayed no effect on basal expression of these genes, they strongly weakened TGF $\beta$-induced gene expression, indicating that NDs affect cell metastasis via interfering with the activities of TGF $\beta$ signaling (Fig. S6, ESI $\dagger$ ).

Tumor organoids can mimic in vivo tumors better than monolayered cancer cells. We verified the effects of NDs on A549 cell-derived tumor organoids in a 3-dimensional culture system. Single cancer cells can develop into spherical tumor organoids in commercial Matrigel, and the addition of NDs had no effect on their sizes or morphologies. In the presence of the TGF $\beta$ ligand, the spherical tumor organoids became irregular 

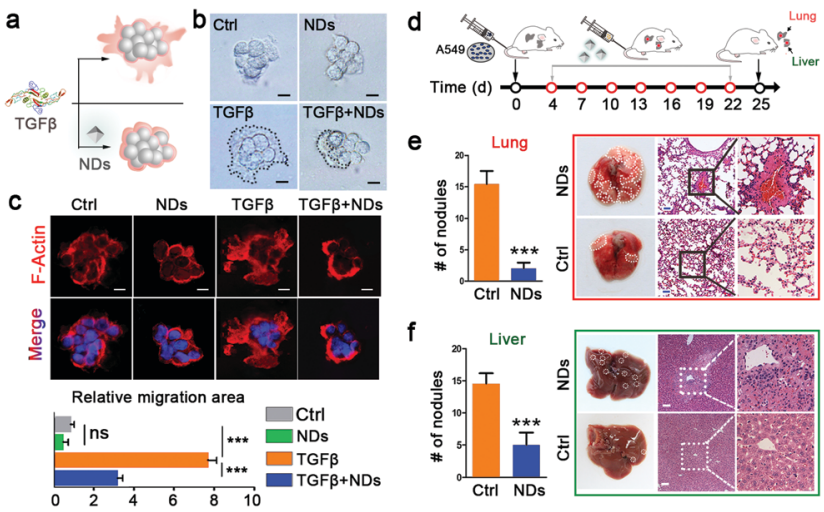

Fig. 4 NDs suppress tumor metastasis in tumor organoids and mice. (a) A scheme for morphological changes in A549-derived tumor organoids. (b and c) The effects of NDs on the morphology of A549-derived tumor organoids are observed by phase contrast microscopy (b) and fluorescence confocal microscopy with quantitation (c). Scale bars, $10 \mu \mathrm{m}$. (d) A scheme for ND treatment in the tumor metastasis model. (e and f) The numbers of tumor nodules in lungs (e) and livers ( $\mathrm{f}$ ) are shown in column charts and indicated with dotted lines in pictures. Data are represented as mean \pm SD $(n=5)$, Student's $t$-test, ${ }^{* *} p<0.001$. Tissue sections are shown by the haematoxylin and eosin (H\&E) staining. Scale bars, $50 \mu \mathrm{m}$.

in shape and displayed lamelipodia-like cytoplasmic extensions. However, NDs remarkably shortened the TGF $\beta$-induced cell extensions and reversed the morphological changes, as revealed by both light and fluorescence microscopies (Fig. 4a-c). There are many well-characterized markers associated with cell metastasis, such as decreased expression of E-cadherin, and increased expression of N-cadherin, fibronectin and Twist. ${ }^{39}$ Our results show that NDs can suppress TGF $\beta$-induced downregulation of E-cadherin and upregulation of $\mathrm{N}$-cadherin, Twist and fibronectin (Fig. S7, ESI $\dagger$ ).

Having observed the effects of NDs on the metastasis of in vitro cultured cancer cells and tumor organoids, we next sought this possibility in xenograft tumors (Fig. 4d). Intravenously injected A549 cells developed numerous metastatic tumor nodules in the lungs and livers in the control mice. We administered NDs ( $80 \mu \mathrm{g}$ per mouse) via the caudal vein every three days for a time window of 18 days, which significantly decreased the number of metastatic tumor nodules in these organs (Fig. 4e and f). A close observation of tissue sections via haematoxylin and eosin (H\&E) staining revealed that the densely proliferated cancer cells severely destroyed the original structure of the lung and liver, and repeated ND injections largely alleviated the tissue lesions (Fig. 4e and f). These data confirm that intravenous administration of NDs inhibits tumor metastasis in vivo.

Lastly, we examined the effects of NDs on the progression of metastatic tumors in the lungs and livers. TAMs are a heterogeneous population of myeloid cells resided in and reprogrammed by the tumor microenvironment, and are generally considered to be closely associated with the malignancy of tumors. ${ }^{40}$ The polarized TAMs are broadly categorized as classically activated, or M1-TAMs, and alternatively activated, or M2-TAMs. M1-TAMs enhance the production of pro-inflammatory cytokines (e.g., TNF $\alpha)$ to boost the immune response for phagocytosis and tumor destruction, whereas M2-TAMs promote anti-inflammatory response, angiogenesis, and tumor progression. ${ }^{41,42}$ The activation of TGF $\beta$ signaling has been shown to affect the population of TAMs and increase the ratio of M2/M1 macrophages through which cancer cells can trigger immunosuppressive activities. ${ }^{43}$ In addition, M2-TAMs produce more TGF $\beta$ to form a positive feedback loop. ${ }^{44}$ In contrast, the blockade of TGF $\beta$ signaling promotes M1-TAM polarization. ${ }^{45}$ We utilized fluorescence-activated cell sorting (FACS) analysis to measure the levels of TAMs and M2-TAMs in the tumor tissues (Fig. 5a). CD11b and F4/80 are cell surface markers of TAMs ${ }^{46,47}$ and CD206 is the surface marker of M2 macrophages. ${ }^{48,49}$ The flow cytometric analysis showed that ND treatment significantly decreased the percentage of TAMs in infiltrated immune cells, and drastically reduced the ratio of M2 macrophages in total TAMs (Fig. 5a-c). Similar results were also observed when we monitored the levels of infiltrated TAMs and M2-TAMs in tumor tissue sections via immunohistofluorescence staining (Fig. 5d, e and Fig. S8, ESI $\dagger$ ).

The polarization between M1 and M2 macrophages is also accompanied by changes in the levels of other protein markers (Fig. S9a, ESI $\dagger$ ). Compared to M1-TAMs, M2-TAMs produce higher amounts of Chitinase 3-like protein 3 (YM-1), but express lower levels of inducible nitric oxide synthase (iNOS). ${ }^{50,51}$ We observed that the administration of NDs strongly boosted the protein levels of iNOS, while severely weakening the expression of YM-1 in the tumor tissues from the lungs and

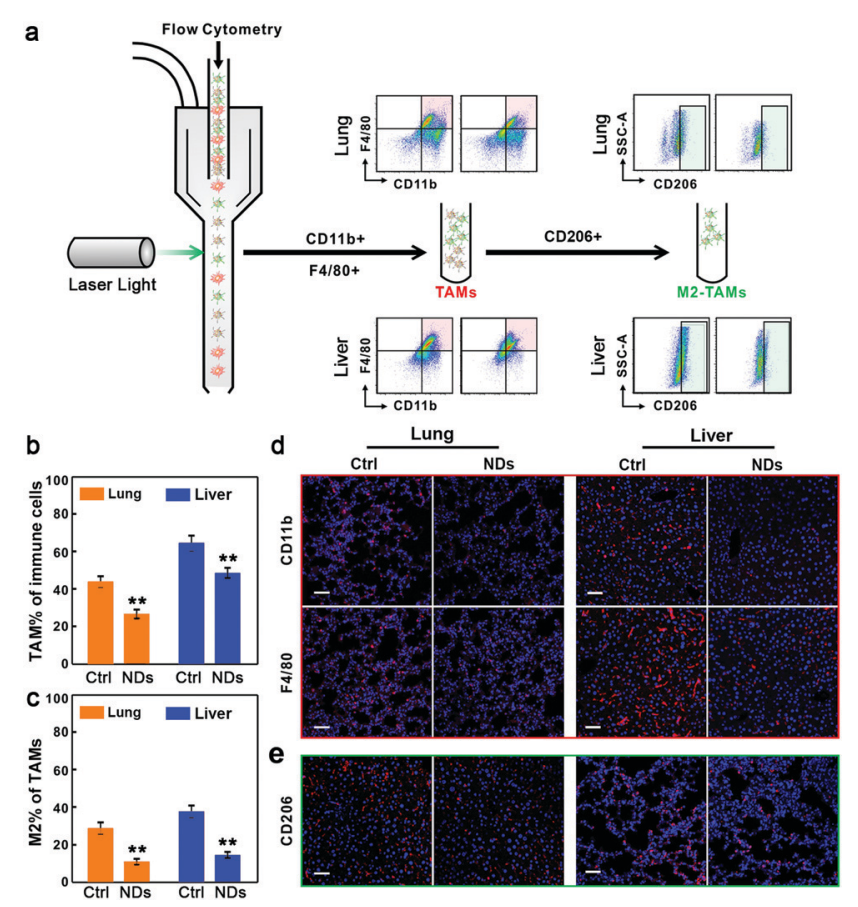

Fig. 5 NDs reduce TAM recruitment and M2 macrophage polarization. (a) FACS analysis of TAMs and M2-TAMs in metastatic tumors. (b) The percentage of TAMs in isolated immune cells in the lung and liver samples. (c) The percentage of M2-TAMs in isolated TAMs. Data are represented as mean $\pm \mathrm{SD}\left(n=3\right.$ ), Student's $t$-test, ${ }^{\star *} p<0.01$. (d and e) Immunofluorescence images of TAMs (d) and M2-TAMs (e) in tissue sections. See also Fig. S8 (ESI $\dagger$ ) for complete data. Scale bars, $50 \mu \mathrm{m}$. 
livers (Fig. S9b and S10, ESI $\dagger$ ). Together, these results confirm the effects of NDs on reducing TAM recruitment and M2 macrophage polarization.

\section{Conclusions}

In this study, we depict the ND interactome network in cancer cells via a proteomic strategy, and further analyze the biological responses of NDs-T $\beta$ RII interaction in several pathological contexts. Our results indicate that the adsorption to NDs promotes lysosomal degradation of TGF $\beta$ receptors and weakens the transduction of TGF $\beta$ signaling. Consequently, cellular uptake or intravenous administration of NDs inhibits the invasiveness and metastasis in cultured cancer cells, tumor organoids and xenograft tumors. In addition, we find that ND injection also dramatically reduces the recruitment of TAMs and M2 macrophage polarization, thereby alleviating the phenotypes of tumor metastasis in mice. Given that NDs have been extensively applied as a type of biocompatible nanocarrier in biomedical research, our study implies more profound utilization of NDs for cancer combination therapy.

\section{Experimental section}

\section{Cell culture}

Human lung cancer A549 cells were obtained from the Cell Bank of the Chinese Academy of Sciences and cultured in Dulbecco's Modified Eagle Medium (DMEM) or Roswell Park Memorial Institute (RPMI) 1640 medium supplemented with 10\% fetal bovine serum (FBS), at $37{ }^{\circ} \mathrm{C}$ in a humidified incubator with $5 \% \mathrm{CO}_{2}$.

\section{Pull-down assay and mass spectrometry analysis}

The A549 cells were spun down from the medium, extensively washed with phosphate buffer saline (PBS), and treated with a lysis buffer (20 mM Na $2 \mathrm{HPO}_{4}, 20 \mathrm{mM} \mathrm{NaH}_{2} \mathrm{PO}_{4}, \mathrm{pH} 7.4,150 \mathrm{mM}$ $\mathrm{NaCl}, 2 \mathrm{mM}$ ethylenediaminetetraacetic acid (EDTA), $0.5 \mathrm{mM}$ $\mathrm{Na}_{3} \mathrm{VO}_{4}, 0.6 \%$ Nonidet P-40, $0.5 \mathrm{mM}$ dithiothreitol, protease inhibitors cocktail and phosphatase inhibitors cocktail). The cleared supernatant was incubated with NDs for $4 \mathrm{~h}$ at $4{ }^{\circ} \mathrm{C}$ on a rotator. The ND-protein complexes were washed three times with the washing buffer $\left(20 \mathrm{mM} \mathrm{Na} \mathrm{HPO}_{4}, 20 \mathrm{mM} \mathrm{NaH} \mathrm{PO}_{4}\right.$, pH 7.4, $150 \mathrm{mM} \mathrm{NaCl}, 2 \mathrm{mM}$ EDTA, $0.5 \mathrm{mM} \mathrm{Na}_{3} \mathrm{VO}_{4}, 0.25 \%$ Nonidet P-40, $0.5 \mathrm{mM}$ dithiothreitol and protease inhibitors cocktail), and three times with the washing buffer excluding detergent and protease inhibitors. The bound proteins on the ND surface were eluted with $5 \%$ formic acid. Trypsin digestion was carried out overnight. Samples were purified by reversephase C-18 chromatography and analyzed on a Lineal Trap Quadropole (LTQ) ion trap mass spectrometer. The mass spectrometry data were searched against the UniProt human database to identify candidate proteins.

\section{Cytotoxicity assay}

The cytotoxicity of NDs in A549 cells was measured with Cell Counting Kit-8 (CCK-8, Sigma-Aldrich). A549 cells were seeded in a 96-well plate at a density of $5 \times 10^{3}$ cells per well and incubated with NDs of various concentrations for the indicated time. After washing with PBS, $10 \mu \mathrm{L}$ of the CCK-8 solution was added to each well of the plate and incubated for $2 \mathrm{~h}$ at $37^{\circ} \mathrm{C}$. The absorbance at $450 \mathrm{~nm}$ was detected using a microplate reader (Bio-Rad 680).

\section{Western blotting and co-localization analysis}

To examine the effects of NDs on the levels of cellular proteins, A549 cells were incubated with NDs for $3 \mathrm{~h}$ and cultured for an additional $16 \mathrm{~h}$ before sample collection. The following antibodies were used. Rabbit anti-T $\beta$ RI and rabbit anti-T $\beta$ RII were from Santa Cruz Biotechnology. Rabbit anti-Smad2 and rabbit anti-Smad3 were obtained from Cell Signaling Technology. Rabbit anti-Lrp6 was from Abclone Technology. Goat anti-rabbit IgG-HRP, goat anti-mouse IgG-HRP and mouse anti-Tubulin were from Abcam. To probe the subcellular localizations of TGF $\beta$ receptors, A549 cells were transfected with RFP-tagged Lamp1. On the next day, the cells were treated with NDs for $3 \mathrm{~h}$ and cultured for an additional $16 \mathrm{~h}$. The cells were then fixed with $4 \%$ paraformaldehyde, permeabilized with $0.5 \%$ Triton X-100 in PBS, and blocked with $5 \%$ bovine serum albumin. After incubation with the anti-T $\beta$ R1 or anti-T $\beta$ RII antibody at $4{ }^{\circ} \mathrm{C}$ overnight, the cells were washed with PBS, incubated with fluorescent secondary antibody (goat anti-rabbit Alexa Fluor 488, Thermo Fisher Scientific), and stained with 4',6-diamidino-2-phenylindole (DAPI). The co-localizations of T $\beta$ R1 or T $\beta$ RII with Lamp1 were imaged with a confocal laser scanning microscope (510 NLO, Zeiss) and quantified with ImageJ software.

\section{Wound-healing migration assay and transwell invasion assay}

In the wound-healing assay, an A549 cell monolayer was scratched using a sterile $200 \mu \mathrm{L}$ pipette tip after incubating with $100 \mu \mathrm{g} \mathrm{mL}^{-1} \mathrm{NDs}$ for $3 \mathrm{~h}$. The cells were washed three times with PBS, and cultured in RPMI 1640 medium supplemented with $2 \%$ FBS in the absence or presence of $5 \mathrm{ng} \mathrm{mL}{ }^{-1}$ TGF $\beta$ (MCE, HY-P7118). Phase contrast microscopy images were taken at 0 and $36 \mathrm{~h}$. The migration rates were quantified using the ImageJ software. In the transwell invasion assay, A549 cells $\left(2 \times 10^{4}\right)$ treated with $100 \mu \mathrm{g} \mathrm{mL}{ }^{-1}$ NDs for $3 \mathrm{~h}$ were cultured in RPMI 1640 medium containing 2\% FBS in the upper well of the Costar Transwell System (Corning). The bottom chamber was filled with $700 \mu \mathrm{L}$ RPMI 1640 medium supplemented with 10\% FBS in the absence or presence of $5 \mathrm{ng} \mathrm{mL}{ }^{-1}$ TGF $\beta$ ligand. After $36 \mathrm{~h}$ induction, non-migrated cells on the upper surface of the chamber were removed with a cotton swab. Cells that migrated into the lower chamber were fixed with $4 \%$ paraformaldehyde and stained with crystal violet. The images were recorded by light microscopy and the number of migrated cells was quantified by ImageJ software.

\section{Tumor organoid culture and invasion assay}

For the 3D tumor organoid invasion assay, 4000 cells were preincubated with $100 \mu \mathrm{g} \mathrm{mL}{ }^{-1}$ NDs for $3 \mathrm{~h}$, and were seeded into 8-well chamber slides (Thermo Fisher Scientific) coated with Matrigel ( $70 \mu \mathrm{L}$ per well, Corning) in the presence or absence of the TGF $\beta$ ligand. Fresh medium was replenished every 2 days. 
Four days later, the cells were fixed with $4 \%$ paraformaldehyde and stained with Alexa Fluor 568 conjugated phalloidin (Thermo Fisher Scientific) for $3 \mathrm{~h}$. Nuclei were visualized by DAPI staining (Invitrogen) and the actin microfilament was monitored by confocal microscopy (510 NLO, Zeiss).

\section{Real-time PCR}

Total RNAs from A549 cells were isolated with TriZol (Ambion) and reverse-transcribed into complementary DNA using the ReverTraAce qPCR RT Kit (TOYOBO). The relative levels of gene transcripts compared to the control gene actin were determined by quantitative real-time PCR, which was performed with SYBR Green Realtime PCR Master Mix (TOYOBO) in the StepOne Real-Time PCR System (Applied Biosystems).

\section{Animals and treatment}

To establish the cancer metastasis model, cultured A549 cells were trypsinized, centrifuged (1400 rpm, $4 \mathrm{~min}$ ), washed twice in PBS, and injected $\left(2 \times 10^{3}\right.$ cells in $\left.100 \mu \mathrm{L} \mathrm{PBS}\right)$ through the caudal vein of 7-8 week old nude mice (day 0 ). Intravenous administration of NDs ( $80 \mu \mathrm{g}$ per mouse) was performed on day 4 , and was repeated every 3 days until day 22 . On day 25 , the animals were sacrificed. The lungs and livers were collected and fixed in 4\% PFA, dehydrated, embedded in paraffin, and sectioned using standard methodologies. Tissues were stained with haematoxylin and eosin (H\&E), and evaluated by light microscopy (PerkinElmer). All mouse experiments were reviewed and approved by the Animal Care and Use Committee of Shanghai Institute of Nutritional Sciences, Chinese Academy of Sciences.

\section{Flow cytometry}

Tumor nodules on the lungs and livers were excised and dissociated into single-cell suspensions using the Miltenyi Tumor Dissociation Kit for murine tissues (130-096-730). Samples were filtered through $70 \mu \mathrm{m}$ cell strainers. For flow cytometry profiling, anti-CD16/CD32 (eBioscience, 14-0161-82), APC conjugated anti-CD45 (eBioscience, 47-04510-82), FITC conjugated anti-CD11b (eBioscience, 11-0112-82), PE conjugated anti-F4/80 (eBioscience, 12-4801-80) and BV421 conjugated anti-CD206 (Biolegend, 141717) were incubated with cells in PBS containing 5\% FBS for 45 min on ice. Cell sorting was performed with a BD FACS Aria and the results were analyzed by the FlowJo software (Tree Star).

\section{Conflicts of interest}

The authors declare no competing financial interests.

\section{Acknowledgements}

This study was supported by the National Natural Science Foundation of China (31571498 and 81773434), China Postdoctoral Science Foundation (2018M633027 and 2018M632135) and the Major Science and Technology Innovation Program of Shanghai Municipal Education Commission (2018YFC1705103).

\section{Notes and references}

1 X. Michalet, F. F. Pinaud, L. A. Bentolila, J. M. Tsay, S. Doose, J. J. Li, G. Sundaresan, A. M. Wu, S. S. Gambhir and S. Weiss, Science, 2005, 307, 538-544.

2 T. Wang, L. Wang, X. Li, X. Hu, Y. Han, Y. Luo, Z. Wang, Q. Li, A. Aldalbahi, L. Wang, S. Song, C. Fan, Y. Zhao, M. Wang and N. Chen, ACS Appl. Mater. Interfaces, 2017, 9, 18619-18625.

3 Y. P. Han, X. M. Li, H. B. Chen, X. J. Hu, Y. Luo, T. Wang, Z. J. Wang, Q. Li, C. H. Fan, J. Y. Shi, L. H. Wang, Y. Zhao, C. F. Wu and N. Chen, ACS Appl. Mater. Interfaces, 2017, 9, 21200-21208.

4 P. Horcajada, T. Chalati, C. Serre, B. Gillet, C. Sebrie, T. Baati, J. F. Eubank, D. Heurtaux, P. Clayette, C. Kreuz, J. S. Chang, Y. K. Hwang, V. Marsaud, P. N. Bories, L. Cynober, S. Gil, G. Ferey, P. Couvreur and R. Gref, Nat. Mater., 2010, 9, 172-178.

5 Y. Wang, F. H. Wang, Y. Y. Shen, Q. J. He and S. R. Guo, Mater. Horiz., 2018, 5, 184-205.

6 Z. J. Wang, W. H. Liu, J. Y. Shi, N. Chen and C. H. Fan, Mater. Horiz., 2018, 5, 344-362.

7 Z. Wang, Y. Fu, Z. Kang, X. Liu, N. Chen, Q. Wang, Y. Tu, L. Wang, S. Song, D. Ling, H. Song, X. Kong and C. Fan, J. Am. Chem. Soc., 2017, 139, 15784-15791.

8 Y. Ji, Y. Xiao, L. Xu, J. He, C. Qian, W. Li, L. Wu, R. Chen, J. Wang, R. Hu, X. Zhang, Z. Gu and Z. Chen, Adv. Sci., 2018, 5, 1700867.

9 N. L. Rosi, D. A. Giljohann, C. S. Thaxton, A. K. Lytton-Jean, M. S. Han and C. A. Mirkin, Science, 2006, 312, 1027-1030.

10 J. H. Park, L. Gu, G. von Maltzahn, E. Ruoslahti, S. N. Bhatia and M. J. Sailor, Nat. Mater., 2009, 8, 331-336.

11 L. Han, J. M. Xia, X. Hai, Y. Shu, X. W. Chen and J. H. Wang, ACS Appl. Mater. Interfaces, 2017, 9, 6941-6949.

12 X. Li, L. Song, X. Hu, C. Liu, J. Shi, H. Wang, L. Zhan and H. Song, ACS Appl. Mater. Interfaces, 2018, 10, 3449-3458.

13 F. Zhao, Y. Zhao, Y. Liu, X. Chang, C. Chen and Y. Zhao, Small, 2011, 7, 1322-1337.

14 Y. Miao, J. Xu, Y. Shen, L. Chen, Y. Bian, Y. Hu, W. Zhou, F. Zheng, N. Man, Y. Shen, Y. Zhang, M. Wang and L. Wen, ACS Nano, 2014, 8, 6131-6144.

15 H. Y. Yi, Z. Y. Wang, X. J. Li, M. Yin, L. H. Wang, A. Aldalbahi, N. N. El-Sayed, H. Wang, N. Chen, C. H. Fan and H. Y. Song, Theranostics, 2016, 6, 1810-1820.

16 N. Chen, H. Wang, Q. Huang, J. Li, J. Yan, D. N. He, C. H. Fan and H. Y. Song, Small, 2014, 10, 3603-3611.

17 Y. Zhang, Z. Y. Wang, X. J. Li, L. Wang, M. Yin, L. H. Wang, N. Chen, C. H. Fan and H. Y. Song, Adv. Mater., 2016, 28, 1387-1393.

18 J. Whitlow, S. Pacelli and A. Paul, J. Controlled Release, 2017, 261, 62-86.

19 J. I. Chao, E. Perevedentseva, P. H. Chung, K. K. Liu, C. Y. Cheng, C. C. Chang and C. L. Cheng, Biophys. J., 2007, 93, 2199-2208.

20 D. R. Tasat, M. E. Bruno, M. Domingo, P. Gurman, O. Auciello, M. L. Paparella, P. Evelson, M. B. Guglielmotti and D. G. Olmedo, J. Biomed. Mater. Res., Part B, 2017, 105, 2408-2415. 
21 F. Zhang, Q. Song, X. Huang, F. Li, K. Wang, Y. Tang, C. Hou and H. Shen, ACS Appl. Mater. Interfaces, 2016, 8, 1087-1097.

22 V. Vaijayanthimala, P. Y. Cheng, S. H. Yeh, K. K. Liu, C. H. Hsiao, J. I. Chao and H. C. Chang, Biomaterials, 2012, 33, 7794-7802.

23 N. Mohan, C. S. Chen, H. H. Hsieh, Y. C. Wu and H. C. Chang, Nano Lett., 2010, 10, 3692-3699.

24 D. G. Lim, N. Rajasekaran, D. Lee, N. A. Kim, H. S. Jung, S. Hong, Y. K. Shin, E. Kang and S. H. Jeong, ACS Appl. Mater. Interfaces, 2017, 9, 31543-31556.

25 V. N. Mochalin, O. Shenderova, D. Ho and Y. Gogotsi, Nat. Nanotechnol., 2012, 7, 11-23.

26 A. E. Nel, L. Madler, D. Velegol, T. Xia, E. M. V. Hoek, P. Somasundaran, F. Klaessig, V. Castranova and M. Thompson, Nat. Mater., 2009, 8, 543-557.

27 C. D. Walkey and W. C. W. Chan, Chem. Soc. Rev., 2012, 41, 2780-2799.

28 M. Mahmoudi, I. Lynch, M. R. Ejtehadi, M. P. Monopoli, F. B. Bombelli and S. Laurent, Chem. Rev., 2011, 111, 5610-5637.

29 C. D. Walkey, J. B. Olsen, F. Y. Song, R. Liu, H. B. Guo, D. W. H. Olsen, Y. Cohen, A. Emili and W. C. W. Chan, ACS Nano, 2014, 8, 2439-2455.

30 C. Q. Yi, C. C. Fong, Q. Zhang, S. T. Lee and M. S. Yang, Nanotechnol., 2008, 19, 095102.

31 S. S. Karajanagi, A. A. Vertegel, R. S. Kane and J. S. Dordick, Langmuir, 2004, 20, 11594-11599.

32 H. Y. Yi, X. J. Li, Z. Y. Wang, M. Yin, L. H. Wang, A. Aldalbahi, N. N. El-Sayed, H. Wang, N. Chen, L. N. Chen, C. H. Fan and H. Y. Song, Part. Part. Syst. Charact., 2017, 34, 1600208.

33 M. I. Setyawati, C. Y. Tay, S. L. Chia, S. L. Goh, W. Fang, M. J. Neo, H. C. Chong, S. M. Tan, S. C. Loo, K. W. Ng, J. P. Xie, C. N. Ong, N. S. Tan and D. T. Leong, Nat. Commun., 2013, 4, 1673.

34 Y. F. Huang, H. P. Liu, X. L. Xiong, Y. Chen and W. H. Tan, J. Am. Chem. Soc., 2009, 131, 17328-17334.

35 N. Chen, Y. P. Han, Y. Luo, Y. F. Zhou, X. J. Hu, Y. Yu, X. D. Xie, M. Yin, J. L. Sun, W. Y. Zhong, Y. Zhao, H. Y. Song and C. H. Fan, Mater. Horiz., 2018, 5, 1204-1210.

36 P. Franzen, P. Tendijke, H. Ichijo, H. Yamashita, P. Schulz, C. H. Heldin and K. Miyazono, Cell, 1993, 75, 681-692.
37 J. L. Wrana, L. Attisano, J. Carcamo, A. Zentella, J. Doody, M. Laiho, X. F. Wang and J. Massague, Cell, 1992, 71, 1003-1014. 38 X. Hu, X. Li, M. Yin, P. Li, P. Huang, L. Wang, Y. Jiang, H. Wang, N. Chen, C. Fan and H. Song, ACS Appl. Mater. Interfaces, 2017, 9, 18575-18583.

39 L. Wang, Z. J. Wang, X. M. Li, Y. Zhang, M. Yin, J. Li, H. Y. Song, J. Y. Shi, D. S. Ling, L. H. Wang, N. Chen and C. H. Fan, Nano Res., 2018, 11, 2746-2755.

40 Y. Qian, S. Qiao, Y. F. Dai, G. Q. Xu, B. L. Dai, L. S. Lu, X. Yu, Q. M. Luo and Z. H. Zhang, ACS Nano, 2017, 11, 9536-9549.

41 X. G. Li, W. B. Yao, Y. Yuan, P. Z. Chen, B. Li, J. Q. Li, R. A. Chu, H. Y. Song, D. Xie, X. Q. Jiang and H. Wang, Gut, 2017, 66, 157-167.

42 S. Zanganeh, G. Hutter, R. Spitler, O. Lenkov, M. Mahmoudi, A. Shaw, J. S. Pajarinen, H. Nejadnik, S. Goodman, M. Moseley, L. M. Coussens and H. E. Daldrup-Link, Nat. Nanotechnol., 2016, 11, 986-994.

43 T. J. Standiford, R. Kuick, U. Bhan, J. Chen, M. Newstead and V. G. Keshamouni, Oncogene, 2011, 30, 2475-2484.

44 X. Z. Ye, S. L. Xu, Y. H. Xin, S. C. Yu, Y. F. Ping, L. Chen, H. L. Xiao, B. Wang, L. Yi, Q. L. Wang, X. F. Jiang, L. Yang, P. Zhang, C. Qian, Y. H. Cui, X. Zhang and X. W. Bian, J. Immunol., 2012, 189, 444-453.

45 A. Sica, A. Saccani, B. Bottazzi, N. Polentarutti, A. Vecchi, J. van Damme and A. Mantovani, J. Immunol., 2000, 164, 762-767.

46 D. G. DeNardo, J. B. Barreto, P. Andreu, L. Vasquez, D. Tawfik, N. Kolhatkar and L. M. Coussens, Cancer Cell, 2009, 16, 91-102.

47 W. Zhang, X. D. Zhu, H. C. Sun, Y. Q. Xiong, P. Y. Zhuang, H. X. Xu, L. Q. Kong, L. Wang, W. Z. Wu and Z. Y. Tang, Clin. Cancer Res., 2010, 16, 3420-3430.

48 C. R. Zhang, X. H. Yu, L. Q. Gao, Y. Zhao, J. H. Lai, D. H. Lu, R. Bao, B. Jia, L. J. Zhong, F. Wang and Z. F. Liu, Theranostics, 2017, 7, 4276-4288.

49 Q. Z. Zhang, W. R. Su, S. H. Shi, P. Wilder-Smith, A. P. Xiang, A. Wong, A. L. Nguyen, C. W. Kwon and A. D. Le, Stem Cells, 2010, 28, 1856-1868.

50 B. Z. Qian and J. W. Pollard, Cell, 2010, 141, 39-51.

51 A. Mantovani and A. Sica, Curr. Opin. Immunol., 2010, 22, 231-237. 